\title{
Solitary fibrous tumor/hemangiopericytoma: treatment results based on the 2016 WHO classification
}

\author{
${ }^{*}$ Kyoung Su Sung, MD,1 Ju Hyung Moon, MD, 2,3 Eui Hyun Kim, MD, PhD, 2,3,6 \\ Seok-Gu Kang, MD, PhD, ,3,6 Se Hoon Kim, MD, PhD, ,3,46 Chang-Ok Suh, MD, PhD, , \\ Sun Ho Kim, MD, PhD, ${ }^{2,3,6}$ Kyu-Sung Lee, MD, PhD, ${ }^{6,7}$ Won Seok Chang, MD, ${ }^{2}$ and \\ Jong Hee Chang, MD, PhD2,3,6
}

\begin{abstract}
1'Department of Neurosurgery, Dong-A University Hospital, Dong-A University College of Medicine, Busan; Departments of ${ }^{2}$ Neurosurgery, ${ }^{4}$ Pathology, and ${ }^{5}$ Radiation Oncology, Severance Hospital, Yonsei University College of Medicine; ${ }^{3}$ Brain Tumor Center and ${ }^{6}$ Brain Research Institute, Yonsei University Health System, Yonsei University College of Medicine; and ${ }^{7}$ Department of Neurosurgery, Brain Tumor Center, Gangnam Severance Hospital, Yonsei University College of Medicine, Seoul, Republic of Korea
\end{abstract}

OBJECTIVE Solitary fibrous tumor/hemangiopericytoma (SFT/HPC) is a new combined entity for which a soft-tissuetype grading system, ranging from grades I to III, has been introduced in the 2016 WHO classification of tumors of the CNS. The results of the treatment of this new disease entity require evaluation.

METHODS The authors retrospectively reevaluated the pathological findings and medical records of patients with SFT/ HPC. This study included 60 patients (27 men and 33 women, median age 42.5 years, range 13-69 years) treated at Severance Hospital between February 1981 and February 2016. Four, 40, and 16 patients were categorized as having SFT/HPC grades I, II, and III, respectively. Among these patients, SFTs diagnosed in 7 patients were regraded as grades I $(n=4), I I(n=2)$, and III $(n=1)$.

RESULTS The median overall survival (OS) was 73.2 months (range 1.4-275.7 months), and the progression-free survival (PFS) after the first operation was 53.8 months (range 1.4-217.7 months). Six patients (10\%) showed extracranial metastasis during a median period of 103.7 months (range 31.9-182.3 months). Nineteen patients (31.7\%) presented with tumor recurrences. The patients in the grade III group had shorter PFS and OS, as well as a shorter period to extracranial metastasis, compared with patients in the grade II group. In the grade II group, patients who underwent gross-total resection showed longer PFS than those who underwent subtotal resection; however, there was no difference in OS. Patients who underwent adjuvant radiation therapy (RT) after surgery had longer PFS compared with that of patients who did not undergo adjuvant RT.

CONCLUSIONS The SFT/HPC grade I group showed a relatively benign course compared with those of the other groups. The grade III group presented a course with a more aggressive nature than that of the grade II group. In the grade II group, the extent of resection and adjuvant RT was significantly associated with longer PFS. The long-term follow-up and periodic systemic evaluation are mandatory to detect systemic metastasis.

https://thejns.org/doi/abs/10.3171/2017.9.JNS171057

KEY WORDS solitary fibrous tumor; hemangiopericytoma; WHO classification; central nervous system; gross-total resection; subtotal resection; oncology

$\mathrm{S}$ TOUT and Murray first described hemangiopericytomas (HPCs) in $1942 .{ }^{25} \mathrm{HPCs}$ are rare, aggressive mesenchymal tumors. In the CNS, HPCs constitute $2.5 \%$ of meningeal tumors and less than $1 \%$ of intracranial tumors. ${ }^{13}$ Most radiological features are similar to those typically associated with meningiomas, ${ }^{6}$ but CNS HPCs have a high propensity for local recurrence and extracranial metastasis, unlike meningiomas. ${ }^{35}$

Solitary fibrous tumors (SFTs) were reported with the first detailed histological description of a pleural mass in

ABBREVIATIONS EOR = extent of resection; GKS = Gamma Knife surgery; GTR = gross-total resection; HPC = hemangiopericytoma; OS = overall survival; PFS = progression-free survival; RT = radiation therapy; SFT = solitary fibrous tumor; STR = subtotal resection.

SUBMITTED April 27, 2017. ACCEPTED September 25, 2017.

INCLUDE WHEN CITING Published online March 9, 2018; DOI: 10.3171/2017.9.JNS171057.

* W.S.C. and J.H.C. contributed equally to this work and share senior authorship. 
TABLE 1. Demographic characteristics of all 60 patients

\begin{tabular}{lc}
\hline \multicolumn{1}{c}{ Characteristic } & Value \\
\hline Median age in yrs (range) & $42.5(13-69)$ \\
\hline Sex (M/F) & $27 / 33$ \\
\hline 2016 WHO grade (I/I//II) & $4 / 40 / 16$ \\
\hline Median OS in mos (range) & $73.2(1.4-275.7)$ \\
\hline Median PFS in mos (range) & $53.8(1.4-217.7)$ \\
\hline No. w/ RT (adjuvant Tx/after recurrence) & $44(39 / 5)$ \\
\hline No. w/ GTR (\%) & $40(66.7)$ \\
\hline GKS (adjuvant Tx/after recurrence) & $14(6 / 8)$ \\
\hline No. w/ whole-body PET-CT & 37 \\
\hline No. w/ recurrence after initial Tx (\%) & $19(31.7)$ \\
\hline No. w/ reop after recurrence & 13 \\
\hline No. w/ extracranial metastasis (\%) & $6(10)$ \\
\hline Median duration to metastasis in mos (range) & $103.7(31.9-182.3)$ \\
\hline Tx modality after a 2nd or more recurrence & Repeat GKS (12 \\
& patients), repeat \\
& RT (1 patient) \\
\hline
\end{tabular}

$\mathrm{Tx}=$ treatment.

1870 by Wagner. ${ }^{12}$ Since SFTs in the CNS were first presented in $1996,{ }^{3}$ CNS SFTs have been typically considered to be benign (WHO grade I tumors). ${ }^{11}$ However, their clinical behaviors were found to be unpredictable, with several reports of a malignant clinical course..$^{15}$

There is an ongoing debate among sarcoma pathologists about how best to differentiate SFTs and HPCs, owing to the frequent misdiagnosis of HPCs as SFTs, and vice versa. ${ }^{12,30}$ Recent work has shown that the majority of SFTs and HPCs in soft tissue share inversions at 12q13, fusing the NAB2 and STAT6 genes,${ }^{11}$ thus, the term "HPC" is no longer used and these 2 diseases have been integrated into the single term "SFT." Accordingly, after many studies on CNS HPCs and SFTs, they were integrated into a new entity, "solitary fibrous tumor/hemangiopericytoma (SFT/HPC)," in the 2016 WHO classification of tumors of the CNS. ${ }^{24}$

Because of the rarity of this new entity and the recent introduction of the WHO grading scheme, we report our 35 -year experience in treating 60 patients with SFT/HPC at our institution.

\section{Methods}

\section{Study Population and Data Collection}

Patients with SFTs and HPCs treated at our institution (Yonsei University, Severance Hospital) between February 1981 and March 2016 were included in this study (Table 1). The histopathological findings were verified to confirm the diagnosis of SFTs and HPCs by electron microscopy and immunohistochemical staining for CD34, CD99, and STAT6. The presence of CD34 and CD99 was confirmed immunohistochemically at the time of diagnosis. The STAT6 immunohistochemical staining of previous paraffin blocks of pathological slides was performed for this study by an experienced neuropathologist (S.H.K.). We also retrospectively reviewed the medical records of these patients to extract the relevant information. The informa- tion included details on patient age and sex, pathological results, radiological findings, extent of resection (EOR), adjuvant therapy, postoperative complications, local or distant recurrence, and extracranial metastasis at the most recent follow-up. The EOR was quantified as gross-total resection (GTR) or subtotal resection (STR). EOR was established from the operative records and was defined as "gross total" after confirmation using available postoperative imaging, such as MRI. Postoperative adjuvant therapies such as external beam radiation therapy and Gamma Knife surgery (GKS) were performed within 6 months after the initial surgery.

In the 2007 WHO CNS classification, HPCs were listed as either WHO grade II or III tumors, with criteria for the diagnosis of anaplastic HPC as WHO grade III that included high mitotic activity ( $>5$ per hpf) and/or necrosis and at least 2 of the following features: hemorrhage, moderate to high nuclear atypia, and high cellularity. ${ }^{23}$ SFTs were not graded in the 2007 WHO CNS classification and were generally believed to be benign (WHO grade I tumors) ${ }^{23}$ However, $10 \%$ of SFTs showed more aggressive behavior, local recurrence, and distant metastasis. . $^{16,17,39}$ Based on the pathological results, such as mitotic index, cellularity, necrosis, and nuclear atypia, we categorized our patients with SFT/HPC into those with WHO grade I, grade II, or grade III tumors according to the 2016 WHO classification system for CNS tumors. ${ }^{24}$ For the purpose of this study, an experienced neuropathologist (S.H.K.) independently reviewed the pathological slides.

Progression-free survival (PFS) was calculated from the time of the first operation to the date of the confirmation of local recurrence and extracranial metastasis. Local recurrence was defined as definite evidence supporting tumor growth in the operative site in follow-up postoperative imaging. Extracranial metastasis was deemed to be present if symptomatic or asymptomatic lesions in extraCNS sites were confirmed using imaging studies such as PET-CT, CT, and MRI. The period to metastasis was defined as the time from the first operation to the confirmation of extracranial metastasis. The overall survival (OS) was calculated as the time from the initial surgery to death or the date of the last postoperative follow-up.

\section{Statistical Analysis}

The demographic characteristics of the patients in each group were compared using independent 2 -sample t-tests and chi-square tests for continuous and categorical variables, respectively. The survival curves for each group were plotted using the Kaplan-Meier method and compared using log-rank (Mantel-Cox) tests. All statistical analyses were performed using SPSS software (version 18.0 KO, SPSS Korea), with $\mathrm{p}<0.05$ considered statistically significant.

\section{Results \\ Patient Population}

Patient characteristics, including age, sex, pathological grade, EOR, adjuvant treatment, recurrence, and extracranial metastasis, are summarized in Table 1. A total of 60 patients with SFT/HPC were treated at our institu- 
TABLE 2. Comparison of the HPC and SFT groups (previously diagnosed)

\begin{tabular}{lcc}
\hline \multicolumn{1}{c}{ Variable } & HPC $(n=53)$ & SFT $(n=7)$ \\
\hline Median age in yrs (range) & $42.5(13-69)$ & $43(33-68)$ \\
\hline Sex (M/F) & $25 / 28$ & $2 / 5$ \\
\hline 2016 WHO grade (I/II/III) & $0 / 38 / 15$ & $4 / 2 / 1$ \\
\hline No. w/ GTR (\%) & $36(67.9)$ & $4(57.1)$ \\
\hline No. w/ RT (adjuvant Tx/after recurrence) & $41(37 / 4)$ & $3(2 / 1)$ \\
\hline No. w/ GKS (adjuvant Tx/after recurrence) & $12(5 / 7)$ & $2(1 / 1)$ \\
\hline No. w/ recurrence (\%) & $18(34.0)$ & $1(14.3)$ \\
\hline No. w/ extracranial metastasis (\%) & $6(11.3)$ & $0(0)$ \\
\hline
\end{tabular}

tion between February 1981 and March 2016. The median age of the 27 men and 33 women was 42.5 years (range 13-69 years). The pathological diagnosis of SFT/HPC was made by identifying typical features, such as branching "staghorn" vasculature, widespread reticulin staining, absence of epithelial membrane antigen staining, electron microscopic imaging, and immunohistochemical staining of CD34, CD99, and STAT6. Based on the 2016 WHO criteria, 4,40 , and 16 patients were classified as having grade I, II, and III SFT/HPC tumors, respectively. All patients underwent resection. In total, 40 patients $(66.7 \%)$ underwent GTR and 20 (33.3\%) underwent STR.

From 1981 to 2003, we treated 22 patients. Among them, 11 patients received adjuvant radiation therapy (RT). Since then, we have increasingly recognized the importance of adjuvant therapy for HPC. Therefore, adjuvant therapy was performed for all 38 patients treated since 2004 , except 4 patients who were classified in the grade I group. Of these 34 patients, 28 received adjuvant RT and 6 received GKS. GKS was performed only for remnant lesions after STR. However, adjuvant RT was the main adjuvant treatment modality and was performed regardless of the EOR in our institution. The adjuvant RT was administered to 65\% (39/60) of patients following the first operation, regardless of the EOR, and the median dose was 5940 cGy (range 5000-6600 cGy, 1.8-2.2 Gy/fraction, 23-33 fractions). Repeat RT was performed on only 1 patient who received adjuvant RT and showed intracranial seeding; the dose was $5880 \mathrm{cGy}$ (2.1 Gy, 28 fractions). Adjuvant GKS was performed on any remnant tumor after STR and was administered to only 6 patients. The median marginal dose was 15 Gy (range 13-15.5 Gy). In addition, GKS was performed on 8 patients who did not receive adjuvant GKS and was administered for small recurrent lesions that did not show definite intracranial seeding, with a median marginal dose of 15 Gy (range 14-17.5 Gy). The total number of GKS treatments administered to these patients was 26 . The median marginal dose was $15 \mathrm{~Gy}$ (range 14-18 Gy).

Patients were followed up by the neurooncology teams in their respective clinics. All patients visited the hospital within 3 months of surgery and had regular outpatient clinic visits with annual follow-up brain imaging. The recurrence rate after initial treatment in our series was $31.7 \%$ (19/60). We performed 12 repeat GKSs and 1 repeat RT for a second or more recurrence. The extracranial
TABLE 3. Comparison of the GTR and STR groups for WHO grade II

\begin{tabular}{lccr}
\hline \multicolumn{1}{c}{ Variable } & GTR $(\mathrm{n}=27)$ & STR $(\mathrm{n}=13)$ & $\mathrm{p}$ Value \\
\hline Median age (yrs) & 41.5 & 42 & 0.263 \\
\hline Median PFS (mos) & 126.6 & 53.4 & $<0.001$ \\
\hline Median OS (mos) & 201.9 & 136.5 & 0.315 \\
\hline Sex (M/F) & $13 / 14$ & $7 / 6$ & 0.736 \\
\hline No. w/ recurrence (\%) & $8(29.6)$ & $8(61.5)$ & 0.054 \\
\hline No. w/ extracranial metastasis & 5 & 0 & \\
\hline
\end{tabular}

metastasis rate was $10 \%(6 / 60)$ with a median period to metastasis of 103.7 months (range 31.9-182.3 months).

\section{Patients Previously Diagnosed With SFT}

CNS SFT was first diagnosed in 2005, and subsequently, 7 patients with CNS SFT have been treated at our institution (Table 2). In the SFT group, 4, 2, and 1 patients were classified as having grade I, II, and III tumors, respectively. There was 1 case of recurrence and no cases of extracranial metastasis or death. The follow-up period of the SFT group was shorter than that of the HPC group. We did not perform a statistical analysis between the 2 groups because it no longer had any clinical meaning after the new WHO classification.

\section{WHO Groups}

\section{SFT/HPC Grade I}

There were 4 patients in the WHO grade I group. In this group, there were no recurrences or extracranial metastases, with a median follow-up period of 42.9 months (range 14.9-78.6 months). We did not perform adjuvant RT because the pathological results showed no mitosis, high cellularity, or nuclear atypia.

\section{SFT/HPC Grade II}

At the first operation in the WHO grade II group, GTR was achieved in 27 patients $(67.5 \%)$ and STR was performed in 13 patients (32.5\%; Table 3). The GTR group included 8 patients $(29.6 \%)$ with a recurrence and had a relatively lower recurrence rate than that of the STR group $(\mathrm{n}=8,61.5 \%)$. However, the difference in recurrence rates was not statistically significant $(\mathrm{p}=0.054)$. In contrast, the median PFS of the GTR group was 126.6 months, significantly longer than that of the STR group (53.4 months, $\mathrm{p}<$ $0.001)$. The 1-, 5-, and 10-year recurrence rates of the GTR group were $0 \%, 5.3 \%$, and $44.7 \%$, while the 1 - and 5 -year recurrence rates in the STR group were $8.3 \%$ and $68.6 \%$, respectively ( $p<0.001 ;$ Fig. 1 left). The OS graph did not present a significant difference between the 2 groups $(\mathrm{p}=$ 0.315; Fig. 1 right).

Twenty-five patients in the WHO grade II group underwent postoperative adjuvant RT. This group showed significant advantages in PFS compared with that of the group treated without adjuvant RT ( $p=0.025$; Fig. 2 left). The 1-, 5-, 10-, and 15-year recurrence rates in the group treated with adjuvant RT were $0 \%, 0 \%, 55.5 \%$, and $70.3 \%$, respectively, while the 1-, 5-, and 10-year recurrence rates 

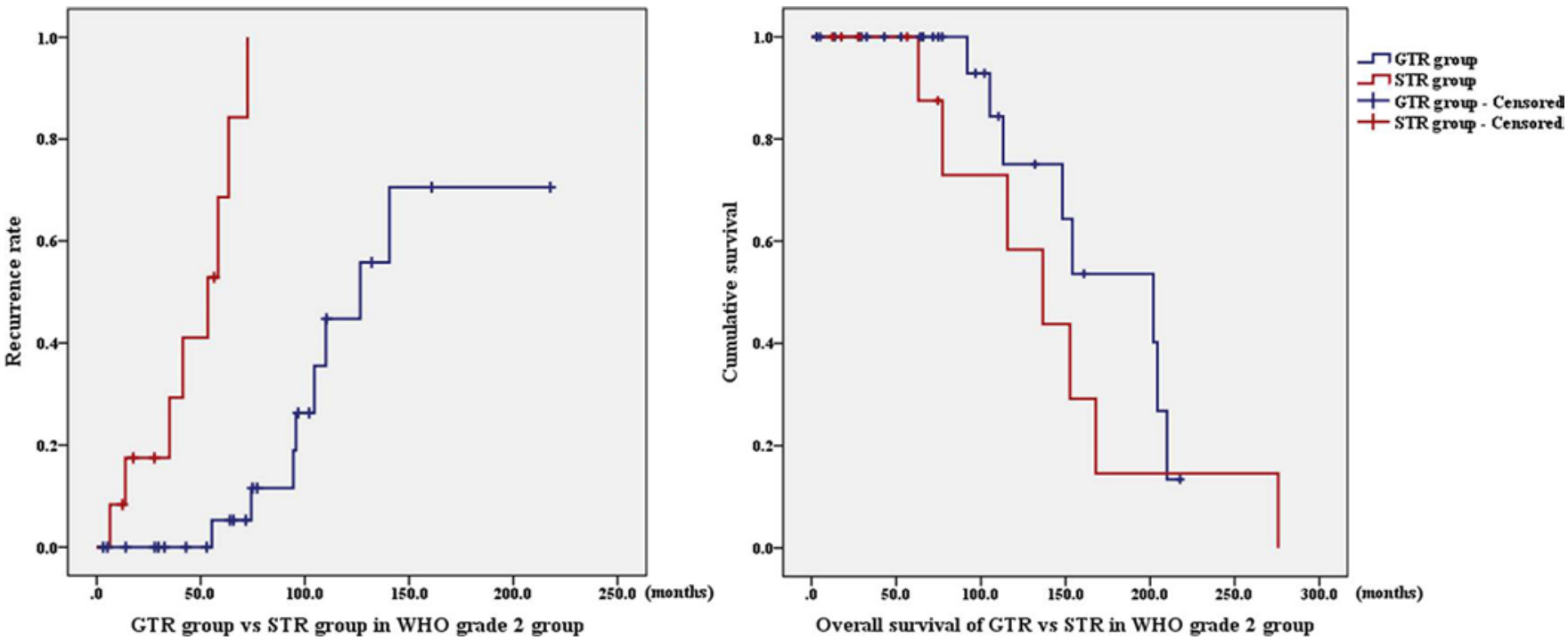

FIG. 1. Kaplan-Meier survival curves showing a comparison of recurrence rate and OS between the GTR $(n=27)$ and STR $(n=$ 13) groups in patients with WHO grade II tumors. Left: The STR group showed a statistically significant higher recurrence rate compared with the GTR group ( $p=0.00$, log-rank test). Right: Comparison of OS between the 2 resection groups showing no statistical significance $(p=0.315)$. Figure is available in color online only.

in the group treated without adjuvant RT were $7.7 \%, 55.1 \%$, and $73.1 \%$, respectively. Patients undergoing adjuvant RT had no significant advantage in OS over the group treated without adjuvant RT ( $\mathrm{p}=0.946$; Fig. 2 right). The 1-, 5-, $10-$, and 20-year survival rates in the group treated with adjuvant RT were $100 \%, 100 \%, 59.3 \%$, and $19.8 \%$, respectively, while the 1-, 5-, 10-, and 20-year survival rates in the group treated without adjuvant RT were $100 \%, 100 \%$, $74.1 \%$, and $11.6 \%$, respectively. In the adjuvant RT group, the main causes of death were extracranial metastasis in 2 patients and systemic infections in 3 patients associated with neurological morbidity due to intracranial recurrence. The causes of deaths in the nonadjuvant group were 1 case of extracranial metastasis, 4 cases of intracranial seeding, 1 case of intraventricular hemorrhage, 1 case of remnant tumor bleeding, 2 cases of meningitis, and 1 case of systemic infection related to recurrence.

Extracranial metastases occurred in 5 patients (12.5\%)
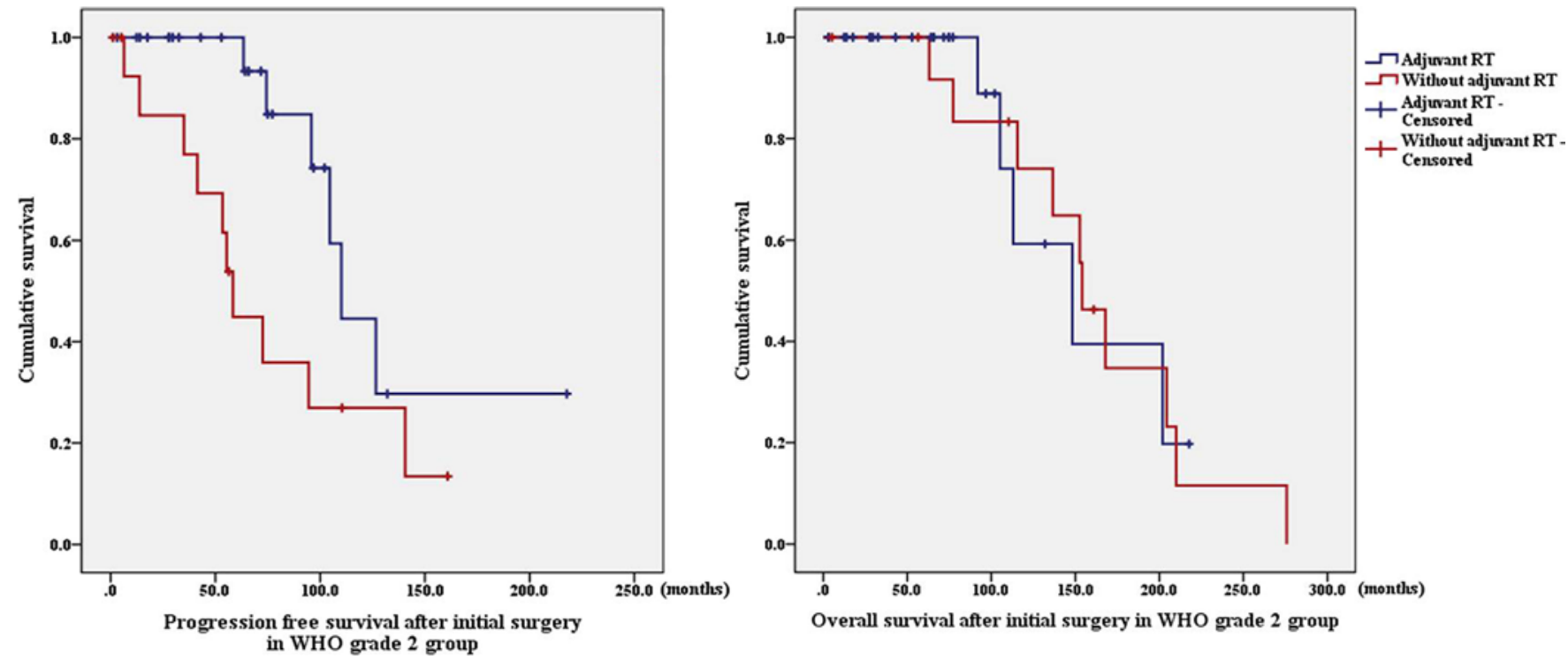

FIG. 2. Kaplan-Meier survival curves showing a comparison of OS and PFS between initial surgery with adjuvant RT group $(n=25)$ and without adjuvant RT group $(n=15)$ in patients with WHO grade II tumors. Left: The surgery with adjuvant RT group showed a statistically significant longer PFS compared with the surgery without adjuvant RT group $(p=0.025$, log-rank test). Right: Comparison of OS between the 2 groups showed no statistical significance $(p=0.946)$. Figure is available in color online only. 
TABLE 4. Comparison of WHO grade II and III groups

\begin{tabular}{lccc}
\hline \multicolumn{1}{c}{ Variable } & Grade II $(\mathrm{n}=40)$ & Grade III $(\mathrm{n}=16)$ & $\mathrm{p}$ Value \\
\hline Median age $(\mathrm{yrs})$ & 42 & 44 & 0.942 \\
\hline Median PFS (mos) & 102.1 & $\mathrm{NR}$ & 0.869 \\
\hline Median OS (mos) & 154.1 & 103.9 & 0.003 \\
\hline No. w/ adjuvant RT (\%) & $25(62.5)$ & $14(87.5)$ & 0.107 \\
\hline $\begin{array}{c}\text { Median duration until } \\
\text { metastasis in mos }\end{array}$ & $111.3(5$ patients) & $31.9(1$ patient) & 0.457 \\
\hline
\end{tabular}

$\mathrm{NR}=$ not reached.

in the WHO grade II group. There were 5 cases of extracranial metastasis in the GTR group and none in the STR group. Intracranial recurrence was noted in only 2 of the 5 patients, and the remaining 3 patients showed extracranial metastasis without local recurrence. Adjuvant RT was performed in 4 of these 5 patients. The median duration until metastasis was 111.3 months (range 74.4-182.3 months).

\section{Comparison of SFT/HPC Grade II and III Groups}

The WHO grade III group showed a more aggressive clinical course compared with that of the WHO grade II group (Table 4). The PFS of the grade III group did not differ significantly from that of the grade II group ( $\mathrm{p}=$ 0.869 ; Fig. 3 left). There was only 1 case of extracranial metastasis in the WHO grade III group. The period to metastasis in this patient was 31.9 months, relatively shorter than that of the patients in the grade II group, although this difference was not significant. The median OS of the grade III group was 103.9 months, significantly shorter than that of the grade II group ( $\mathrm{p}=0.003$; Fig. 3 right). The $1-, 5-, 10-$, and 20 -year survival rates of the grade II group were $100 \%, 100 \%, 69.6 \%$, and $14.8 \%$, respectively, while the 1- and 5-year survival rates of the grade III group were $92.3 \%$ and $71.8 \%$, respectively.

\section{Discussion}

Since their first inclusion in the 2002 WHO classification of CNS tumors, SFTs and HPCs have been considered distinct entities. SFT was classified as a tumor with a benign course, while HPC was considered locally aggressive. ${ }^{22}$ This concept remained unchanged in the 2007 WHO revision, and HPCs were further divided into HPCs and anaplastic HPCs, which were graded as WHO grades II and III. ${ }^{23}$ In early studies on SFTs, some authors reported indolent growth, low rate of recurrence, and almost no extracranial metastases. ${ }^{38}$ However, other authors reported SFTs with an aggressive nature, showing high recurrence rates and extracranial metastasis. ${ }^{28,29}$ These 2 diseases have led to many debates among sarcoma pathologists about how best to differentiate SFTs and HPCs. ${ }^{30}$ Because of the large overlap of histopathological and clinical features and a lack of clear diagnostic criteria, the 2 entities have been frequently misdiagnosed as each other. ${ }^{12}$ However, fusion of the NAB2 and STAT6 genes and the overexpression of STAT6 have been reported in both SFTs and HPCs; ${ }^{4,31}$ therefore, these 2 diseases were unified into a single entity in the 2013 WHO classification of soft tissue and bone. Based on these concepts, several authors have reported that CNS SFTs and HPCs both present with NAB2-STAT6 fusion and immunohistochemical staining of STAT6. $5,11,36,40$

Unlike soft-tissue pathologists, neuropathologists continued to use the term "hemangiopericytoma." Therefore, the 2016 WHO classification of tumors of the CNS created the combined term "SFT/HPC." 24 Based on the SFT/HPC grading scale described in the 2016 WHO classification of CNS, we reviewed the pathological results, including cellularity, mitosis, and nuclear atypia. WHO grades II and
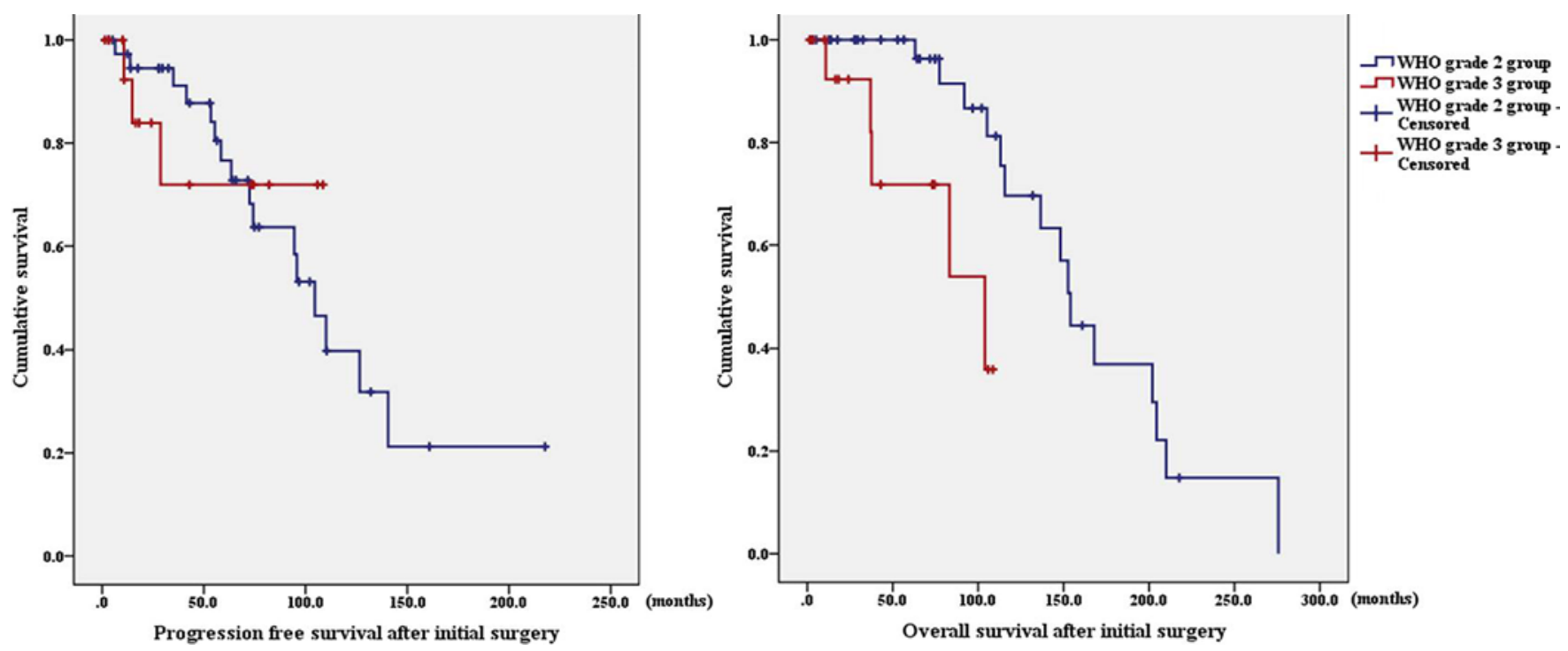

FIG. 3. Kaplan-Meier survival curves showing a comparison between the WHO grade II and III groups. Left: The PFS between the 2 groups showed no statistically significant difference $(p=0.869)$. Right: The OS of the WHO grade III group was significantly shorter than that of the WHO grade II group ( $p=0.003$, log-rank test). Figure is available in color online only. 

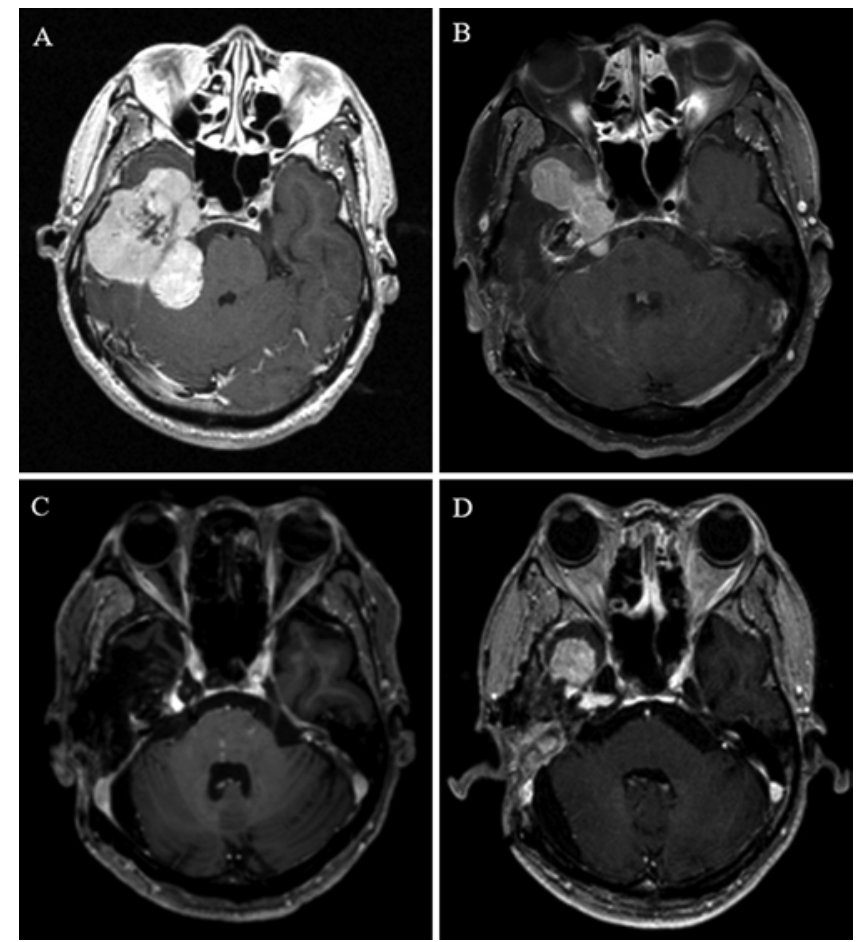

FIG. 4. Axial MR images from a patient in the SFT/HPC WHO grade II group. A: The patient presented with a large tumor at the right-side temporal, petrous bone, cerebellopontine angle area. B: After 107 months of initial treatment, the patient showed a fourth tumor recurrence and underwent RT. C: After the fourth recurrence and RT, the followup imaging showed a good treatment response state. D: After the fifth recurrence of tumor, the patient underwent a fourth GKS.

III were differentiated based on a cutoff of 5 or more mitoses per $10 \mathrm{hpfs}$. The criteria for WHO grade I included no nuclear atypia and no high cellularity, depending on our neuropathologist's determination.

In our study, 7 patients had been previously diagnosed with SFTs. It was first diagnosed in 2005. Therefore, at our institution the mean follow-up period in the SFT group was not long relative to that of the HPC group. Among these 7 patients, 4, 2, and 1 were categorized as having grade I, II, and III tumors, respectively. The SFT cases in our study showed relatively benign and indolent courses, with 1 recurrence, no deaths, and no extracranial metastasis. However, we performed adjuvant RT in 2 patients with high cellularity and a high mitotic index in the pathological results. Therefore, this approach appears to be a proper management even without the presence of an established treatment protocol for SFTs.

Some authors have reported a significant proportion of extracranial metastasis among cases of SFT. ${ }^{15}$ One report compared the clinical characteristics of SFTs to those of HPCs, including patient sex ratio, location, and age at presentation. ${ }^{8}$ However, these clinical and pathological comparisons have produced many debates among pathologists. Furthermore, these comparisons are no longer clinically meaningful.

In previous studies, $85 \%-90 \%$ of SFTs showed a benign, indolent clinical course. ${ }^{8,28,29}$ This group became the SFT/HPC grade I group. Based on the pathologic results, we did not perform adjuvant RT in the grade I group. GTR was achieved in 3 patients (75\%) and STR was performed in $1(25 \%)$. The median follow-up period was 34.3 months (range 14.9-78.6 months); however, longer follow-up periods are necessary to detect unpredictable clinical behavior.

A number of authors have reported a significant correlation between GTR and improved survival. The GTR rates reported in their studies varied between $50 \%$ and 83.3\%., $, 2,10,33,34$ However, these studies did not include WHO grades. In our study, the GTR rate was $66.7 \%$. In the WHO grade II group, the GTR rate was $67.5 \%$. The GTR group had a significantly longer PFS compared with that of the STR group in the WHO grade II group. Moreover, the GTR group had a lower recurrence rate than that of the STR group, although the difference was not significant. However, unlike in the other studies, $1,2,10,33,34$ there was no significant difference in OS between the GTR and STR groups in the WHO grade II group. In addition, patients with adjuvant RT had a significantly longer PFS compared with that of patients without adjuvant RT in the WHO grade II group. Melone et al. and other groups have reported that adjuvant RT prolonged the time before first recurrence; $6,14,20,21,25,33$ our results support those of the previous studies. Another study reported that adjuvant RT had a significant effect on OS. ${ }^{35,37}$ However, our results showed that there was no significant difference in OS between the adjuvant and nonadjuvant RT groups. These results showed the importance of aggressive treatment, including stereotactic radiosurgery and RT after recurrence. Some authors have reported that stereotactic radiosurgery is effective in disease control after recurrence..$^{19}$ Another study showed the importance of multimodal treatment for the recurrence of HPCs. ${ }^{32}$ Although the GTR and adjuvant RT group did not show much benefit for OS compared with the STR and nonadjuvant RT group, these factors can increase the disease-free period of patients. The physicians tried to remove as much tumor during surgery as possible, even if they performed STR. It is important to reduce the patient's tumor burden related to RT as much as possible for effective adjuvant treatment results. Even after STR, adjuvant RT is meaningful to maintain patient's OS similar to that of the GTR group. Moreover, even after recurrence, active treatment options such as reoperation, repeat GKS, and repeat RT are meaningful in maintaining OS. We performed 12 repeat GKSs and 1 repeat RT after a second or more recurrence.

For example, a 51-year-old man had a large tumor in his right-side temporal lobe, petrous bone, and cerebellopontine angle region. The intended second-stage operation and adjuvant GKS for the remnant lesion in the cavernous sinus were performed. The pathologic results indicated an SFT; thus, it was classified as WHO grade II. The patient presented with tumor recurrence at 35 months after the initial treatment. After the second GKS, the patient showed recurrences at 24, 29, 7, and 28 months after each active treatment, respectively. The patient underwent a total of 4 GKS procedures, 1 RT, and 1 reoperation (Fig. 4). The patient remains alive 11.6 years after the initial operation.

The 2007 WHO classification of CNS introduced anaplastic HPCs. Since the introduction of WHO grade III, recent studies have examined the differences in outcomes between the 2 grades of HPC. Some authors reported that 
grade III had a higher recurrence rate compared with that of grade II. ${ }^{7,26,35}$ Damodaran et al. reported that the grade II group had significantly improved survival compared with the grade III group (216 vs 142 months, $\mathrm{p}=0.044$ ). However, in their study, the grade II group showed no significant difference in recurrence. ${ }^{6}$ Their results are similar to those of our study. Our WHO grade III group had a significantly shorter OS than that of the WHO grade II group. However, there was no difference in PFS between these groups. Even before the anaplastic HPC was introduced in 2007, we performed active adjuvant RT for HPC for higher-grade cases based on pathologic results. In this study, $14(87.5 \%)$ of 16 WHO grade III patients underwent adjuvant RT. In comparison, 25 (62.5\%) of $40 \mathrm{WHO}$ grade II patients underwent adjuvant RT. We believe that our treatment strategy for grade III disease resulted in the relatively lower recurrence rate in this group. However, analysis of OS revealed that the WHO grade III group had a more aggressive disease compared with that in the WHO grade II group, with higher disease-related mortality and morbidity; longer follow-up periods are also necessary to detect recurrence in patients in the WHO grade III group.

There has been controversy about extracranial metastasis in SFT/HPC. The overall reported extracranial metastasis rates vary from $2.6 \%$ to $56 \%$. $1,13,15,18,19,26,27,35$ Our extracranial metastatic rate was $10 \%$. Guthrie et al. reported that the development of extracranial metastasis results in a significantly reduced survival period..$^{14}$ In our study, extracranial metastasis was the cause of death in 3 of the 6 patients. The earliest and longest reported periods to metastasis were 7 months and 20 years, respectively. ${ }^{6}$ Our longest period to metastasis was 182.3 months. Moreover, some authors have reported that a higher grade is associated with an increased risk of extracranial metastasis. ${ }^{26}$ However, others have shown that a higher grade and adjuvant RT are not correlated with extracranial metastasis. ${ }^{6,25,35}$ In the current study, extracranial metastasis was not associated with EOR, adjuvant RT, and intracranial local recurrence. These are unpredictable characteristics. Therefore, long-term follow-up and careful clinical examinations to detect extracranial metastasis and to identify its clinical characteristics must be performed at regular intervals. Some authors recommend regular chest radiographs at 6- to 12-month intervals as an inexpensive option, with follow-up CT scans for lung metastasis. ${ }^{26}$ We performed regular checkups using whole-body PET-CT every 5 years. In the current study, 6 patients presented with extracranial metastasis. The metastatic lesion was confirmed by pathological examination in 5 patients. One patient underwent follow-up CT scans at regular intervals because of a very small lesion in the lung, which was first detected through PET-CT.

\section{Conclusions}

Patients in the SFT/HPC WHO grade I group showed a more benign course. The grade III group presented with a disease of a more aggressive nature and shorter OS compared with that of the grade II group. In the grade II group, the EOR and adjuvant RT were significant factors for longer PFS. In addition, aggressive treatment after recurrence is important for patients' survival. The long-term follow- up and periodic systemic evaluation are mandatory to detect delayed recurrence and/or systemic metastasis.

\section{Acknowledgments}

This study was supported by a faculty research grant of Yonsei University College of Medicine (no. 6-2012-0205) and a National Research Foundation of Korea (NRF) grant funded by the Korean government (MSIP no. 2014R1A2A1A10052762).

\section{References}

1. Alén JF, Lobato RD, Gómez PA, Boto GR, Lagares A, Ramos A, et al: Intracranial hemangiopericytoma: study of 12 cases. Acta Neurochir (Wien) 143:575-586, 2001

2. Bassiouni H, Asgari S, Hübschen U, König HJ, Stolke D: Intracranial hemangiopericytoma: treatment outcomes in a consecutive series. Zentralbl Neurochir 68:111-118, 2007

3. Carneiro SS, Scheithauer BW, Nascimento AG, Hirose T, Davis DH: Solitary fibrous tumor of the meninges: a lesion distinct from fibrous meningioma. A clinicopathologic and immunohistochemical study. Am J Clin Pathol 106:217224, 1996

4. Chmielecki J, Crago AM, Rosenberg M, O'Connor R, Walker SR, Ambrogio L, et al: Whole-exome sequencing identifies a recurrent NAB2-STAT6 fusion in solitary fibrous tumors. Nat Genet 45:131-132, 2013

5. Choi J, Park SH, Khang SK, Suh YL, Kim SP, Lee YS, et al: Hemangiopericytomas in the central nervous system: a multicenter study of Korean cases with validation of the usage of STAT6 immunohistochemistry for diagnosis of disease. Ann Surg Oncol 23 (Suppl 5):954-961, 2016

6. Damodaran O, Robbins P, Knuckey N, Bynevelt M, Wong G, Lee G: Primary intracranial haemangiopericytoma: comparison of survival outcomes and metastatic potential in WHO grade II and III variants. J Clin Neurosci 21:1310-1314, 2014

7. Ecker RD, Marsh WR, Pollock BE, Kurtkaya-Yapicier O, McClelland R, Scheithauer BW, et al: Hemangiopericytoma in the central nervous system: treatment, pathological features, and long-term follow up in 38 patients. J Neurosurg 98:1182-1187, 2003

8. Fargen KM, Opalach KJ, Wakefield D, Jacob RP, Yachnis AT, Lister JR: The central nervous system solitary fibrous tumor: a review of clinical, imaging and pathologic findings among all reported cases from 1996 to 2010. Clin Neurol Neurosurg 113:703-710, 2011

9. Fletcher CDM: The evolving classification of soft tissue tumours - an update based on the new 2013 WHO classification. Histopathology 64:2-11, 2014

10. Fountas KN, Kapsalaki E, Kassam M, Feltes CH, Dimopoulos VG, Robinson JS, et al: Management of intracranial meningeal hemangiopericytomas: outcome and experience. Neurosurg Rev 29:145-153, 2006

11. Fritchie KJ, Jin L, Rubin BP, Burger PC, Jenkins SM, Barthelmeß S, et al: NAB2-STAT6 gene fusion in meningeal hemangiopericytoma and solitary fibrous tumor. J Neuropathol Exp Neurol 75:263-271, 2016

12. Gengler C, Guillou L: Solitary fibrous tumour and haemangiopericytoma: evolution of a concept. Histopathology 48:63-74, 2006

13. Ghose A, Guha G, Kundu R, Tew J, Chaudhary R: CNS hemangiopericytoma: a systematic review of 523 patients. Am J Clin Oncol 40:223-227, 2017

14. Guthrie BL, Ebersold MJ, Scheithauer BW, Shaw EG: Meningeal hemangiopericytoma: histopathological features, treatment, and long-term follow-up of 44 cases. Neurosurgery 25:514-522, 1989 
15. Han N, Kim H, Min SK, Paek SH, Park CK, Choi SH, et al: Meningeal solitary fibrous tumors with delayed extracranial metastasis. J Pathol Transl Med 50:113-121, 2016

16. Hasegawa T, Matsuno Y, Shimoda T, Hasegawa F, Sano T, Hirohashi S: Extrathoracic solitary fibrous tumors: their histological variability and potentially aggressive behavior. Hum Pathol 30:1464-1473, 1999

17. Insabato L, Siano M, Somma A, Gentile R, Santangelo M, Pettinato G: Extrapleural solitary fibrous tumor: a clinicopathologic study of 19 cases. Int J Surg Pathol 17:250-254, 2009

18. Kano H, Niranjan A, Kondziolka D, Flickinger JC, Lunsford LD: Adjuvant stereotactic radiosurgery after resection of intracranial hemangiopericytomas. Int J Radiat Oncol Biol Phys 72:1333-1339, 2008

19. Kim BS, Kong DS, Seol HJ, Nam DH, Lee JI: Gamma knife radiosurgery for residual or recurrent intracranial hemangiopericytomas. J Clin Neurosci 35:35-41, 2017

20. Kim JH, Jung HW, Kim YS, Kim CJ, Hwang SK, Paek SH, et al: Meningeal hemangiopericytomas: long-term outcome and biological behavior. Surg Neurol 59:47-54, 2003

21. Kim YJ, Park JH, Kim YI, Jeun SS: Treatment strategy of intracranial hemangiopericytoma. Brain Tumor Res Treat 3:68-74, 2015

22. Kleihues P, Louis DN, Scheithauer BW, Rorke LB, Reifenberger G, Burger PC, et al: The WHO classification of tumors of the nervous system. J Neuropathol Exp Neurol 61:215229,2002

23. Louis DN, Ohgaki H, Wiestler OD, Cavenee WK, Burger PC, Jouvet A, et al: The 2007 WHO classification of tumours of the central nervous system. Acta Neuropathol 114:97-109, 2007

24. Louis DN, Perry A, Reifenberger G, von Deimling A, Figarella-Branger D, Cavenee WK, et al: The 2016 World Health Organization Classification of Tumors of the Central Nervous System: a summary. Acta Neuropathol 131:803-820, 2016

25. Melone AG, D'Elia A, Santoro F, Salvati M, Delfini R, Cantore $\mathrm{G}$, et al: Intracranial hemangiopericytoma-our experience in 30 years: a series of 43 cases and review of the literature. World Neurosurg 81:556-562, 2014

26. Mena H, Ribas JL, Pezeshkpour GH, Cowan DN, Parisi JE: Hemangiopericytoma of the central nervous system: a review of 94 cases. Hum Pathol 22:84-91, 1991

27. Noh SH, Lim JJ, Cho KG: Intracranial hemangiopericytomas: a retrospective study of 15 patients with a special review of recurrence. J Korean Neurosurg Soc 58:211-216, 2015

28. Ogawa K, Tada T, Takahashi S, Sugiyama N, Inaguma S, Takahashi SS, et al: Malignant solitary fibrous tumor of the meninges. Virchows Arch 444:459-464, 2004

29. Osuga T, Hayashi T, Ishiwatari H, Ono M, Yoshida M, Kimura Y, et al: Pancreatic metastasis from a solitary fibrous tumor of the central nervous system. JOP 15:58-62, 2014

30. Park MS, Araujo DM: New insights into the hemangiopericytoma/solitary fibrous tumor spectrum of tumors. Curr Opin Oncol 21:327-331, 2009

31. Robinson DR, Wu YM, Kalyana-Sundaram S, Cao X, Lonigro RJ, Sung YS, et al: Identification of recurrent NAB2STAT6 gene fusions in solitary fibrous tumor by integrative sequencing. Nat Genet 45:180-185, 2013
32. Rutkowski MJ, Bloch O, Jian BJ, Chen C, Sughrue ME, Tihan T, et al: Management of recurrent intracranial hemangiopericytoma. J Clin Neurosci 18:1500-1504, 2011

33. Rutkowski MJ, Jian BJ, Bloch O, Chen C, Sughrue ME, Tihan T, et al: Intracranial hemangiopericytoma: clinical experience and treatment considerations in a modern series of 40 adult patients. Cancer 118:1628-1636, 2012

34. Rutkowski MJ, Sughrue ME, Kane AJ, Aranda D, Mills SA, Barani IJ, et al: Predictors of mortality following treatment of intracranial hemangiopericytoma. J Neurosurg 113:333339,2010

35. Schiariti M, Goetz P, El-Maghraby H, Tailor J, Kitchen N: Hemangiopericy toma: long-term outcome revisited. Clinical article. J Neurosurg 114:747-755, 2011

36. Schweizer L, Koelsche C, Sahm F, Piro RM, Capper D, Reuss DE, et al: Meningeal hemangiopericytoma and solitary fibrous tumors carry the NAB2-STAT6 fusion and can be diagnosed by nuclear expression of STAT6 protein. Acta Neuropathol 125:651-658, 2013

37. Sonabend AM, Zacharia BE, Goldstein H, Bruce SS, Hershman D, Neugut AI, et al: The role for adjuvant radiotherapy in the treatment of hemangiopericytoma: a Surveillance, Epidemiology, and End Results analysis. J Neurosurg 120:300308, 2014

38. Tihan T, Viglione M, Rosenblum MK, Olivi A, Burger PC: Solitary fibrous tumors in the central nervous system. A clinicopathologic review of 18 cases and comparison to meningeal hemangiopericytomas. Arch Pathol Lab Med 127:432-439, 2003

39. Vallat-Decouvelaere AV, Dry SM, Fletcher CD: Atypical and malignant solitary fibrous tumors in extrathoracic locations: evidence of their comparability to intra-thoracic tumors. Am J Surg Pathol 22:1501-1511, 1998

40. Yalcin CE, Tihan T: Solitary fibrous tumor/hemangiopericytoma dichotomy revisited: a restless family of neoplasms in the CNS. Adv Anat Pathol 23:104-111, 2016

\section{Disclosures}

The authors report no conflict of interest concerning the materials or methods used in this study or the findings specified in this paper.

\section{Author Contributions}

Conception and design: Sung. Acquisition of data: Sung, Moon, WS Chang. Analysis and interpretation of data: Sung, SH Kim, WS Chang. Drafting the article: Sung. Critically revising the article: JH Chang, Sung. Reviewed submitted version of manuscript: JH Chang, Sung. Approved the final version of the manuscript on behalf of all authors: JH Chang. Statistical analysis: Sung. Administrative/technical/material support: SH Kim, Suh. Study supervision: JH Chang, EH Kim, Kang, SH Kim, Lee, WS Chang.

\section{Correspondence}

Jong Hee Chang: Severance Hospital, Yonsei University College of Medicine, Seoul, Republic of Korea.changjh@yuhs.ac. 